\title{
Adaptation of Symmetric Positive Semi-Definite Matrices for the Analysis of Textured Images
}

\section{Adib Akl}

Department of Telecommunications, Faculty of Engineering Holy Spirit University of Kaslik (USEK), P.O. Box 446, Jounieh, Lebanon E-mail:adibakl@usek.edu.lb

\begin{abstract}
This paper addresses the analysis of textured images using the symmetric positive semi-definite matrix. In particular, a field of symmetric positive semidefinite matrices is used to estimate the structural information represented by the local orientation and the degree of anisotropy in structured and sinusoid-like textured images. In order to ensure faithful local structure estimation, an adaptive algorithm for the regularization of the extent of gradient fields smoothing is proposed. Results obtained on different texture samples show the strength of the proposed method in accurately representing the local variation of orientations in the underlying textured images, which paves the way towards an accurate analysis of the texture structures.
\end{abstract}

Keywords: Binary Image, Coherence Factor, Gaussian Kernel, Gradient Field, Image Structure, Orientation, Symmetric Positive Semi-Definite Matrix, Textured Images.

\section{Introduction}

In image processing, textured images are defined as spatial arrangements of pixels intensity describing natural phenomena having repeated patterns with a certain amount of randomness [1,2].

The analysis of textured images is a useful area of study in many application domains. It is used in image classification, fingerprint examination, identification of regions of interest and retrieving similar regions in remote sensing imaging [3-6]. It finds solutions too many problems faced in image and video editing and merging and it can be seen as an important tool in medical imaging $[4,7,8]$. In computer graphics, texture analysis is used to model the surface details [2]. In addition, recent researches prove the efficiency of the analysis of textured images in the interpretation of seismic data and in texture synthesis applications [9-18].

Several methods have been proposed for the analysis of textured images. For instance, a Markov Random Field texture modeling method is proposed in [19]. 
This method is based on the estimation of the local conditional probability density function which leads to mathematically capturing the visual characteristics of the texture into a statistical model describing the interactions between pixel values. A level curves extraction algorithm which consists in retrieving the tangent directions and the curvatures of the image sample is proposed in [20]. Portilla and S imoncelli [21] use an over complete complex wavelet transform in order to parameterize the model by a set of statistics corresponding to basic functions at adjacent locations and scales. Xiang et al. [22] use adaptive-fused features and gradient-based edge information in order to track objects in images.

Model-based texture analysis methods consist in building an image model that can be used to describe the texture sample where the model parameters confine the essential local characteristics of the texture. Model-based methods include the autoregressive models [23], Wold models [24], Gabor and wavelet models [25-27], fractal models [28], Markov and Gibbs random fields [29-31], etc. In these methods, the difficulties reside in choosing the decent model for the underlying image and in determining its parameters.

In order to analyze the orientations of the texture patterns, the method proposed in [32] decomposes the image into a flow field, representing the direction of anisotropy, and a residual pattern obtained by describing the image in a coordinate system built from the flow field. As a preprocessing phase for texture synthesis, Peyré [9] proposes a grouplet-based method which assumes that the geometry of the texture model is an orientation flow that follows the texture patterns.

It has been demonstrated that the use of the structural information can help the analysis of textured images, in different application domains, such as image synthesis [14], image inpainting [33] and video compression [34]. The structural information (i.e., the structure layer) of the textured image is commonly analyzed and represented by the symmetric positive semi-definite matrix, also referred to as the structure tensor or the second-moment matrix, carrying information about the local orientation, the degree of anisotropy and the energy of the underlying image $[14,33,34]$. However, the accuracy of this representation raises a crucial enquiry, as it is constrained by the extent of gradient fields smoothing used to compute the matrices. In other words, a decent size of the gradient smoothing kernel is essential to get relevant local structure estimation. This size is usually adjusted for each textured image, after several structure layer computation runs, in order to tune the symmetric positive semi-definite matrix for relevant representation [14].

In this paper, we propose a method allowing to adaptively estimate the convenient Gaussian kernel size used to smooth the gradient fields leading to a relevant symmetric positive semi-definite matrix field. The images under consideration are structured textured images as well as non-structured sinusoid-like textures.

The remainder of this paper is organized as follows. The symmetric positive semi-definite matrix computation is reviewed in Section 2. The proposed algorithm is detailed in Section 3. Practical results are discussed in Section 4. Finally, conclusions and prospects are drawn in Section 5. 


\section{Symmetric positive semi-definite matrix}

The Symmetric Positive Semi-Definite Matrix is a matrix derived from the gradient of a function where the gradient shows the directions of the greatest degree of increase of the scalar field. It allows the local anisotropy around a site of the spatial domain to be analyzed, by summarizing the degree to which these directions are coherent $[35,36]$. In image processing, it is commonly used to represent edge information and to describe local patterns [37, 38].

The field of symmetric positive Semi-definite Matrices SM of an image $I$ is defined as the field of local covariance matrices of the first partial derivatives of $I$, constructed from estimated gradient fields [39]:

$$
\Lambda I=\left[I_{x} I_{y}\right] ; I_{x}=I^{*} \delta_{x}, I_{y}=I^{*} \delta_{y},
$$

where $\delta_{x}$ and $\delta_{y}$ are isotropic Gaussian derivatives kernel and “*” denotes the linear convolution.

SM is then expressed as [14]:

$$
\mathrm{SM}=G^{\sigma} * \Lambda I \Lambda I^{\mathrm{T}}=\left(\begin{array}{ll}
\mathrm{SM}_{x x} & \mathrm{SM}_{x y} \\
\mathrm{SM}_{x y} & \mathrm{SM}_{y y}
\end{array}\right)=\left(\begin{array}{cc}
G^{\sigma} * I_{x}^{2} & G^{\sigma} * I_{x} I_{y} \\
G^{\sigma} * I_{x} I_{y} & G^{\sigma} * I_{y}^{2}
\end{array}\right),
$$

where $G^{\sigma}$ is a Gaussian weighting kernel of standard deviation $\sigma$ and [.] ${ }^{\mathrm{T}}$ is the transpose operator.

In this paper, the structure layer of a textured image $I$ is represented by the field of symmetric positive semi-definite matrices SM which assigns a symmetric positive semi-definite matrix $\operatorname{SM}(z)$ to each site $z$ of $I$.

An eigen decomposition of matrix $\operatorname{SM}(z)$ gives two non-negative eigenvalues $\gamma_{1}(z)$ and $\gamma_{2}(z)$ related to the variations of intensity in the principle direction of the gradients in the vicinity of $z$ and in the orthogonal direction, the directions are respectively provided by the first and second eigenvectors $e_{1}(z)=\left[\begin{array}{ll}e_{1 x}(z) & e_{1 y}(z)\end{array}\right]^{\mathrm{T}}$ and $e_{2}(z)=\left[e_{2 x}(z) e_{2 y}(z)\right]^{\mathrm{T}}[13]:$

$$
\operatorname{SM}(z)=\left(\begin{array}{cc}
e_{1 x}(z) & e_{2 x}(z) \\
e_{1 y}(z) & e_{2 y}(z)
\end{array}\right)\left(\begin{array}{cc}
\gamma_{1}(z) & 0 \\
0 & \gamma_{2}(z)
\end{array}\right)\left(\begin{array}{ll}
e_{1 x}(z) & e_{1 y}(z) \\
e_{2 x}(z) & e_{2 y}(z)
\end{array}\right) .
$$

The symmetric positive semi-define matrix $\operatorname{SM}(z)$ is generally represented by its orientation factor $\varphi(z)$ calculated from the first eigenvector $e_{1}(z)$ associated to $\gamma_{1}(z)$ and varying between $-\pi / 2$ and $\pi / 2[14]$ :

$$
\varphi(z)=\tan ^{-1}\left(\frac{e_{1 y}(z)}{e_{1 x}(z)}\right) .
$$

A relevant indicator based on $\operatorname{SM}(z)$ is the coherence factor [40], also called confidence factor or dispersion indicator, which is obtained from the eigenvalues and varies between 0 and 1 :

$$
\varsigma(z)=\frac{\gamma_{1}(z)-\gamma_{2}(z)}{\gamma_{1}(z)+\gamma_{2}(z)}
$$

It is considered as a measure of the local anisotropy and it characterizes the dispersion of the gradient orientation, i.e., the local variation of the image geometry. 
The energy (or magnitude) of the symmetric positive semi-definite matrix, defined as the sum of its eigenvalues (i.e., the expectation of the amplitude of the squared gradient), is also an essential factor characterizing the dynamics. In other words, the energy at a certain position expresses the local contrast in the vicinity of this position [41].

In the sequel, the field of symmetric positive semi-definite matrices is represented by its local orientations and coherence images. An example of calculation of a field of symmetric positive semi-definite matrices is presented in Figure 1 showing, from upper-left to bottom-right, the "canvas" textured image [42], its field of symmetric positive semi-definite matrices represented by the orientation and coherence images and the palette used to represent the orientations. Note that this palette is used for all the results that follow. All the images are of size $128 \times 128$ and the standard deviation $\sigma$ of the Gaussian weighting kernel $G^{\sigma}$ (Equation (2)) is of 1.5.

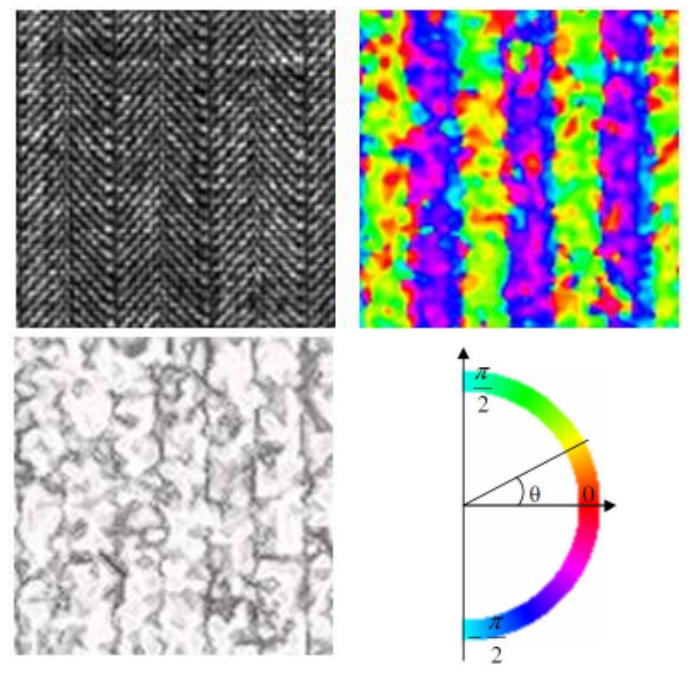

Fig. 1. Example of a field of symmetric positive semi-definite matrices. Upper-left to bottom-right: the "canvas" texture [42], the orientation and coherence images of the matrices field and the palette of orientations

It can be seen in Fig. 1 that the lines oriented at $45^{\circ}$ (or $135^{\circ}$ ) of the texture are detected by the orientation image revealing the structure at the largest scale. On the contrary, the horizontal and vertical stitches of the smallest scale are more difficult to highlight because of the smoothing used to compute the fields of matrices. Moreover, the coherence image shows that the coherence is high within the local regions of approximately same orientations, while the boundaries of those regions, marked by a change in the orientation of the patterns, have a smaller coherence.

\section{Adaptive Gaussian kernel size estimation}

As mentioned earlier, the symmetric positive semi-definite matrix $\operatorname{SM}(z)$ is calculated by gradient field smoothing using a Gaussian weighting kernel $G^{\sigma}$ of 
standard deviation $\sigma$. The spatial smoothing makes $\operatorname{SM}(z)$ more robust to noise and local artifacts. It also allows to spatially distribute the orientation information, mainly available on the strong gradient regions (e.g., edges) [43].

The Gaussian weighting kernel $G^{\sigma}$ is truncated to the interval $[-3 \sigma, 3 \sigma]$, which means that the size of $G^{\sigma}$ can be expressed as

$$
\langle G ^ { \sigma } \rangle = 1 + 2 \longdiv { 3 \sigma }
$$

where " $\ulcorner$ " denotes the rounding up to the nearest integer operator.

Therefore, the choice of $\sigma$ is crucial to get relevant local image analysis. It has to be chosen according to the size of the patterns of the underlying image, in a way to ensure that $G^{\sigma}$ is large enough to faithfully reveal the structure information. Naturally, it should be on the scale of the largest observed pattern, otherwise the structure may be lost [14]. In addition, a high value for $\sigma$ leads to a $\operatorname{smoother} \operatorname{SM}(z)$ and a less local orientation estimation but a better noise robustness. On the contrary, a low $\sigma$ entails an accurate local analysis but with a high sensitivity to noise [44]. This shows the relevance of adaptively estimating a decent size $\left\langle G^{\sigma}\right\rangle$ for the Gaussian kernel $G^{\sigma}$.

The proposed algorithm allows to detect the scale of the largest pattern in the textured image, and therefore to estimate a convenient $\left\langle G^{\sigma}\right\rangle$, which eliminates the need of repetitive SM computations with different values of $\sigma$ in order to reach a faithful local structure representation.

\subsection{Case of structured textures}

In the case of structured textured images (i.e., textures showing regular patterns), the proposed method operates as follows:

- The input texture sample $I$ is transformed to a binary image $I_{\mathrm{b}}$ by means of thresholding operators following the method in [45].

- The position $z_{i}^{\mathrm{c}}=\left(x_{i}^{\mathrm{c}}, y_{i}^{\mathrm{c}}\right)$ of the center $c_{i}$ of each pattern $\operatorname{IP}_{i}\left(i \in\left\{1, \ldots, N_{r}\right\}\right.$; $N_{r}$ is the number of patterns in $I_{\mathrm{b}}$ ) is determined.

- The largest distance $\left(\overrightarrow{\mathrm{IP}}_{i}\right)$ from the center $c_{i}$ of pattern $\operatorname{IP}_{i}\left(i \in\left\{1, \ldots, N_{r}\right\}\right)$ to its borders is calculated.

- The largest pattern, corresponding to the highest $\left(\overrightarrow{\mathrm{IP}}_{i}\right)$, denoted by $\mathrm{IP}_{\max }$ is determined.

- The range of standard deviations which results in a Gaussian kernel having the size of the largest pattern observed in $I_{\mathrm{b}}\left(\left\langle G^{\sigma}\right\rangle=1+2 \mathrm{IP}_{\max }\right)$ is calculated as

$$
R_{P}^{\sigma}=\left[\frac{\mathrm{IP}_{\max }-1}{3}, \frac{\mathrm{IP}_{\max }}{3}\right] .
$$

Therefore, the Gaussian kernel having any standard deviation chosen from $R_{P}^{\sigma}$, is on the scale of the largest pattern in the initial image $I$. Moreover, a value of $\sigma$ which tends to $\mathrm{IP}_{\max } / 3$ is convenient in the case of noisy textured images, in order to ensure a high resistance to noise, while a value of $\sigma$ close to $\left(\mathrm{IP}_{\max }-1\right) / 3$ is more accurate when dealing with noise free textures and leads to a faithful local structure estimation. Fig. 2 shows an illustration of the proposed algorithm. 


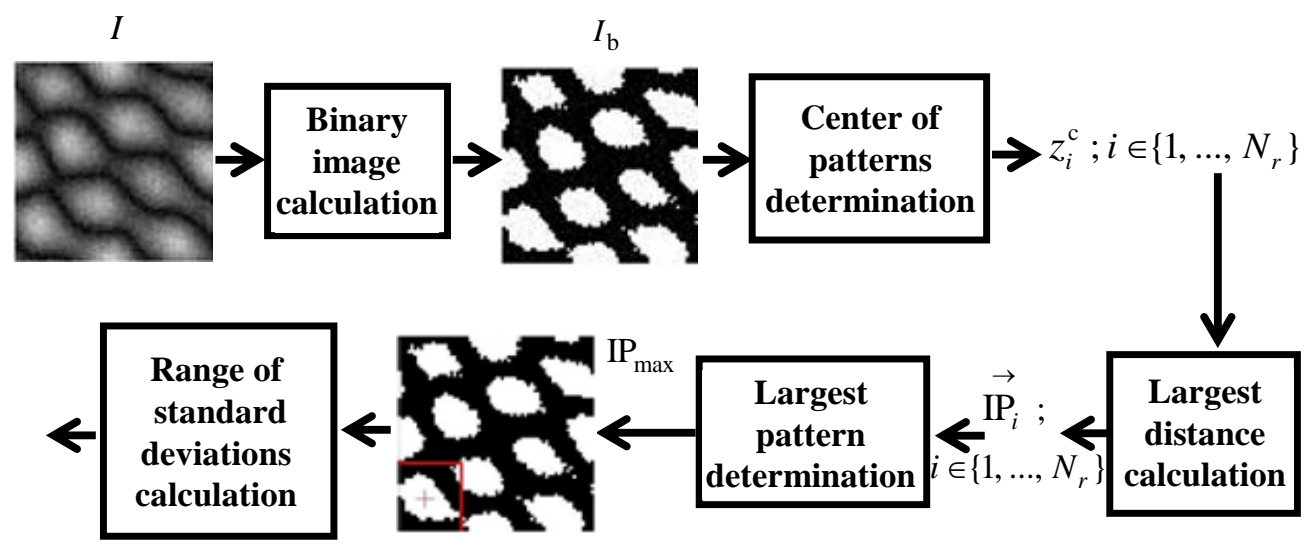

Fig. 2. Illustration of the proposed algorithm. The obtained largest pattern is highlighted in red

\subsection{Case of non-structured sinusoid-like textures}

A non-structured sinusoid-like texture is an image composed of sinusoid waveforms that appear like elongated traces, as it is the case for seismic [46, 47] and laminar [48] textures for example.

When dealing with this type of textured images, the obtained binary image $I_{\mathrm{b}}$ presents an arrangement of laminar structures (Fig. 3). In this case, a modification of the proposed algorithm consists in:

- Detecting the maximum trace width (IT $\mathrm{Imax}_{\text {) }}$ in $I_{\mathrm{b}}$ using four searching directions: vertical, horizontal, first diagonal (i.e., from bottom-left to upper-right) and second diagonal (i.e., from upper-left to bottom-right).

- Computing the acceptable range of standard deviations $\left(R_{T}^{\sigma}\right)$ as in the original algorithm (Section 3.1) while applying $\mathrm{IP}_{\max }=\mathrm{IT}_{\max } / 2$ since $\mathrm{IP}_{\max }$ represents the half size of the Gaussian kernel:

$$
R_{T}^{\sigma}=\left[\frac{\mathrm{IT}_{\max }-2}{6}, \frac{\mathrm{IT}_{\max }}{6}\right]
$$

Fig. 3 illustrates examples of maximum trace width computation on two different sinusoid-like textured images. 

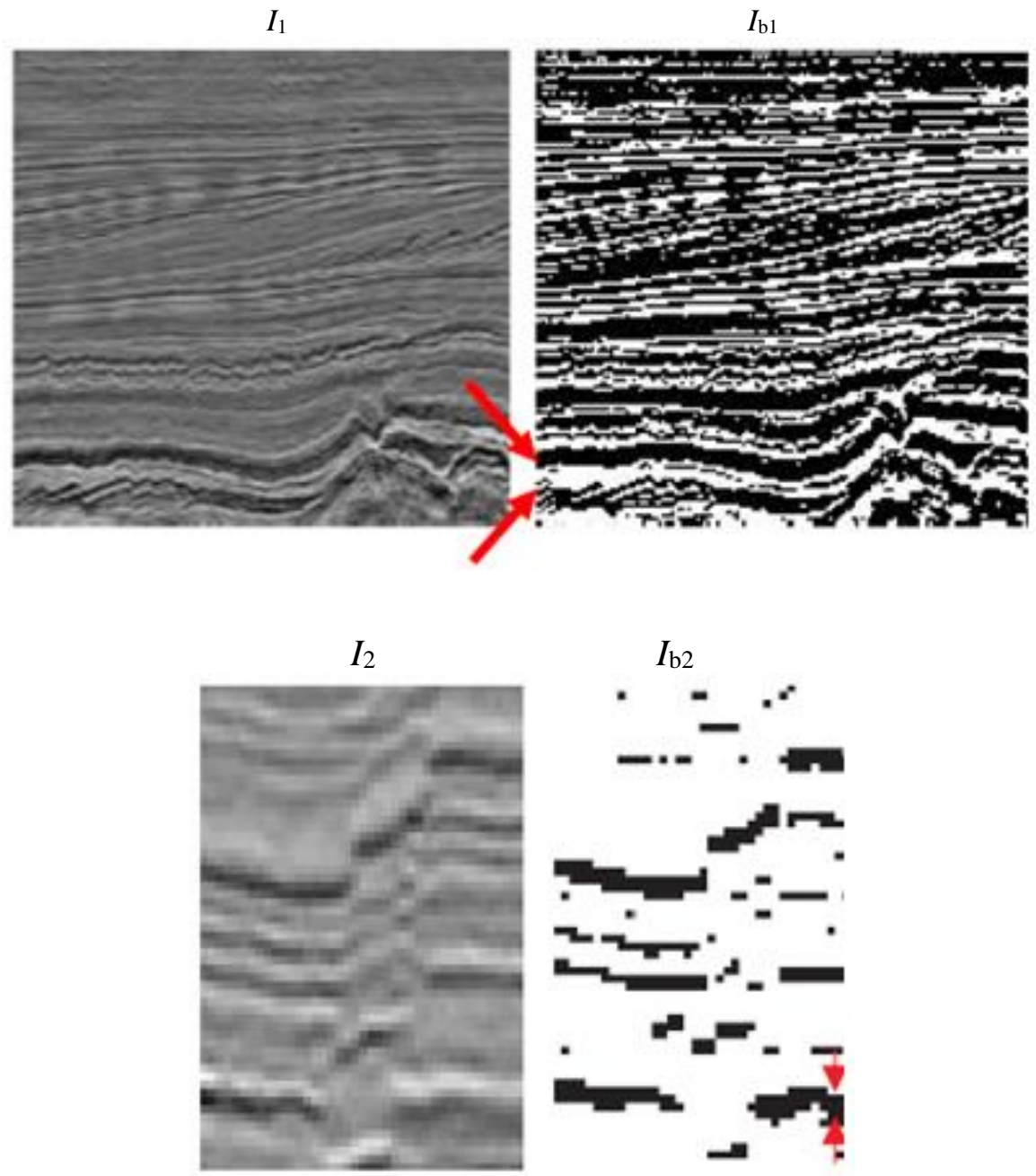

Fig. 3. Non-structured sinusoid-like textures ( $I_{1}$ and $I_{2}$ ) and the corresponding binary images ( $I_{\mathrm{b} 1}$ and $\left.I_{\mathrm{b} 2}\right)$ showing (in red) the detected trace having the largest width

\section{Results}

In this section, the proposed algorithm is evaluated using different texture samples. Fig. 4 presents results obtained on two different structured textures from Brodatz database [42]. The first row shows the input structured textures $\left(I_{1}\right.$ and $\left.I_{2}\right)$ and these 
textures after adding a Gaussian noise of variance $300\left(I_{n_{1}}\right.$ and $\left.I_{n_{2}}\right)$. The second row shows the orientation (left) and coherence (right) images of the field of symmetric positive semi-definite matrices calculated on $I_{n_{1}}$ and $I_{n_{2}}$ using the median value of the acceptable range of standard deviations $\left(R_{P}^{\sigma}\right)$ obtained by the proposed algorithm (Equation (7)). That is $\sigma=2.04$ and $\sigma=2.99$ for textures $I_{n_{1}}$ and $I_{n_{2}}$, respectively. The third to seventh rows show the orientation and coherence images calculated on $I_{n_{1}}$ and $I_{n_{2}}$ with $\sigma=0.5,1.5,2.5,3.5$ and 4.5. All the images are of size $256 \times 256$.

It can be clearly seen that a low standard deviation $(\sigma=0.5)$ leads to a noisy matrix field, with both textures $I_{n_{1}}$ and $I_{n_{2}}$. The standard deviation of 1.5 results in a matrix field which is more resistant to noise. However, the smoothing kernel seems not large enough to faithfully represent the structures of the underlying exemplars, which is highlighted by the distorted patterns present in the orientation and coherence images of the fourth row.

On the other hand, a very high standard deviation $(\sigma=4.5)$ results in oversmoothed fields that fail to represent the structure variations of the input samples. On the contrary, the standard deviations of 2.04 (for texture $I_{n_{1}}$ ) and 2.99 (for texture $I_{n_{2}}$ ), chosen from the range of standard deviations obtained by the proposed algorithm, lead to matrix fields that faithfully represent the structures and local patterns of the exemplars. The resulting orientation and coherence images are noise-free with no over-smoothing effect.

Table 1 presents the Peak Signal-to-Noise Ratio (PSNR) computed between the coherence images obtained with the proposed algorithm on the noisy textures $I_{n_{1}}$ and $I_{n_{2}}$ (Fig. 4) and those obtained with the same algorithm on the noiseless texture samples $\left(I_{1}\right.$ and $\left.I_{2}\right)$.

Table 2 presents the Coefficient of Correlation (CoC) computed between the coherence images obtained with $\sigma=3.5$ and 4.5 and the coherence images obtained using the standard deviation given by the proposed approach (2.04 for $I_{n_{1}}$ and 2.99 for $I_{n_{2}}$ ).

Note that this metric gives information about edge preservation, and therefore, it is used to measure the extent of accurate local analysis.

It can be observed in Tables 1 and 2 that the PSNR increases with the increase of the standard deviation of the Gaussian weighting kernel used to compute the matrix. This verifies that a larger weighting kernel is more resistant to noise and artifacts. In other words, the noise effect on the matrix field becomes more negligible when the standard deviation increases.

On the other hand, the $\mathrm{CoC}$ decreases with the increase of $\sigma$, which expresses a loss in the precision of local structure estimation. This example confirms the tradeoff between the resistivity to noise and the accuracy of local analysis, which highlights the relevance of the proposed approach. 


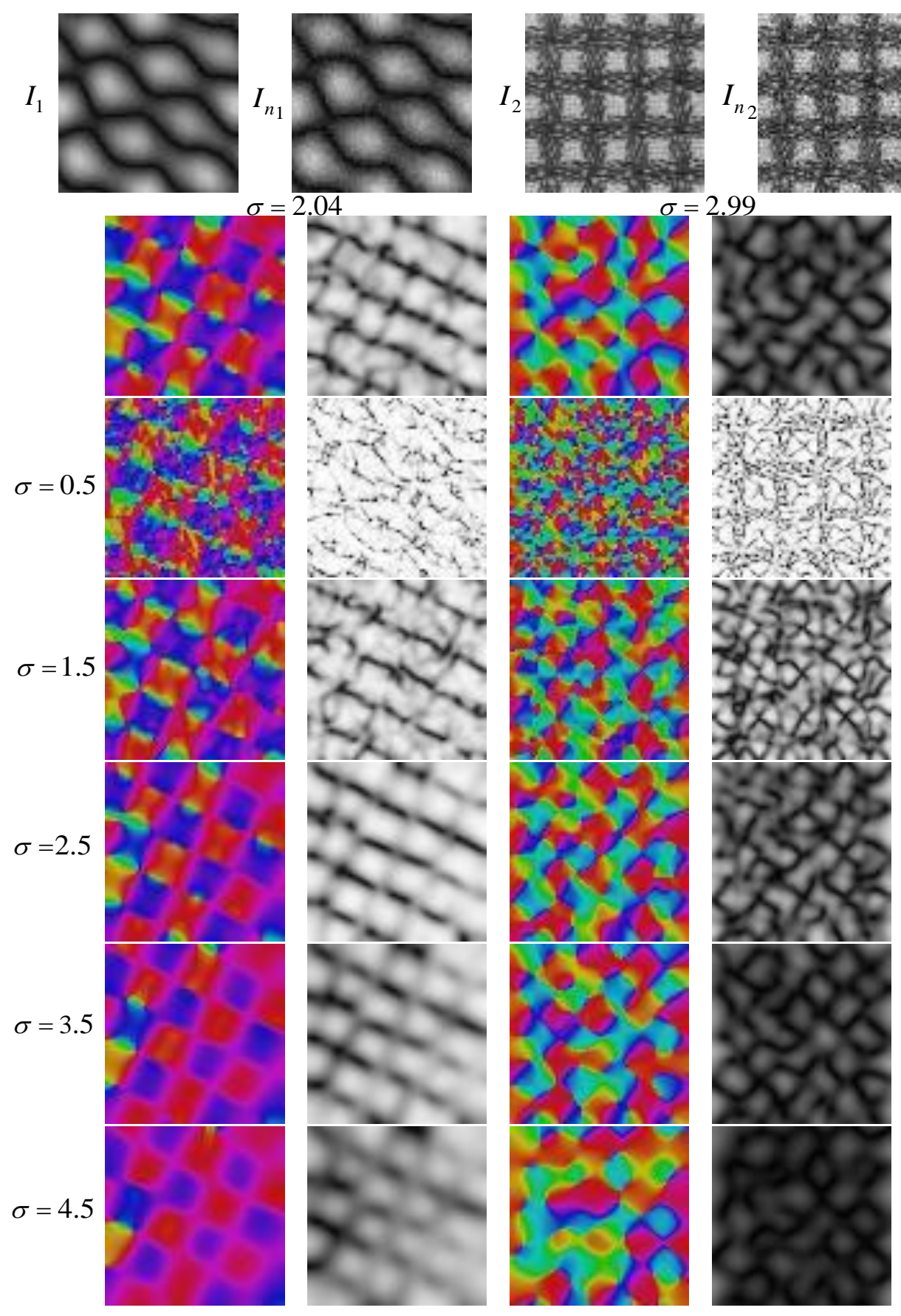

Fig. 4. Results of the proposed algorithm on two structured textures from Brodatz database [42]. 1st row: the original structured exemplars $\left(I_{1}\right.$ and $\left.I_{2}\right)$ and these exemplars after adding a Gaussian noise $\left(I_{n_{1}}\right.$ and $\left.I_{n_{2}}\right)$. 2nd row: orientation (left) and coherence (right) images of the field of symmetric positive semi-definite matrices calculated on $I_{n_{1}}$ and $I_{n_{2}}$ using the standard deviations obtained by the proposed algorithm. 3rd to 7th rows: orientation and coherence images calculated with $\sigma=0.5,1.5,2.5,3.5$ and 4.5 
Table 1. Peak Signal-to-Noise Ratio between the coherence images obtained by the proposed algorithm on $I_{n_{1}}$ and $I_{n_{2}}$ and those obtained with the same algorithm on $I_{1}$ and $I_{2}$ (Fig. 4)

\begin{tabular}{|c|c|c|}
\hline \multirow{2}{*}{ Standard deviation $(\sigma)$} & \multicolumn{2}{|c|}{ PSNR } \\
\cline { 2 - 3 } & $I_{1} / I_{n_{1}}$ & $I_{2} / I_{n_{2}}$ \\
\hline 0.5 & 16.2032 & 17.0124 \\
\hline 1.5 & 17.1154 & 19.2651 \\
\hline 2.5 & 30.1225 & 22.5301 \\
\hline 3.5 & 32.0321 & 25.7587 \\
\hline 4.5 & 33.6514 & 28.1131 \\
\hline
\end{tabular}

Table 2. Coefficient of Correlation between the coherence images obtained with $\sigma=3.5$ and 4.5 , and the coherence images obtained with the proposed approach (Fig. 4)

\begin{tabular}{|c|c|c|}
\hline \multirow{2}{*}{ Standard deviation $(\sigma)$} & \multicolumn{2}{|c|}{ CoC } \\
\cline { 2 - 3 } & $I_{n_{1}}$ & $I_{n_{2}}$ \\
\hline 3.5 & 0.9251 & 0.8984 \\
\hline 4.5 & 0.8801 & 0.7729 \\
\hline
\end{tabular}

Fig. 5 presents results obtained with the proposed algorithm using nonstructured sinusoid-like textures. The first row shows the exemplars $\left(I_{3}, I_{4}\right.$ and $\left.I_{5}\right)$. The second, third and fourth rows show the orientations of the matrix fields calculated using $\sigma=1, \sigma=4$ and the median value of $R_{T}^{\sigma}$ (Equation (8)) obtained by the proposed algorithm; 2.33 for $I_{3}, 3.2$ for $I_{4}$ and 3.12 for $I_{5}$.

In all the results, the standard deviation of 1 leads to a noisy matrix field. As the Gaussian weighting kernel is not large enough to capture the variation of orientations in the sample texture, the orientation images show distorted patterns and artifacts. With textures $I_{3}$ and $I_{4}$, the standard deviation of 4 results in oversmoothed orientation images, while the proposed approach results in orientation images that locally represent the structural variations in the input samples. In other words, the adaptation of the weighting kernel to the scale of the underlying texture patterns leads to a faithful representation of its structure layer. For texture $I_{5}$, the matrix fields obtained with $\sigma=3.12$ and $\sigma=4$ are both of acceptable quality in terms of robustness to noise. However, the proposed method remains advantageous in complexity gain, by eliminating the need of repetitive runs of matrix computation with different values for the standard deviation, in order to obtain an acceptable quality.

The first row of Fig. 6 shows five different input textures and the second row shows the orientations of the matrix fields calculated on these textures using a standard deviation chosen randomly from the acceptable range of standard deviation obtained by the proposed algorithm; $R_{P}^{\sigma}$ (for textures $I_{6}, I_{7}, I_{9}$ and $I_{10}$ ) and $R_{T}^{\sigma}$ (for texture $I_{8}$ ). It can be clearly seen that the obtained fields are artifact-free and they succeed in detecting the variation of orientations in textures $I_{6}$ and $I_{7}$. 

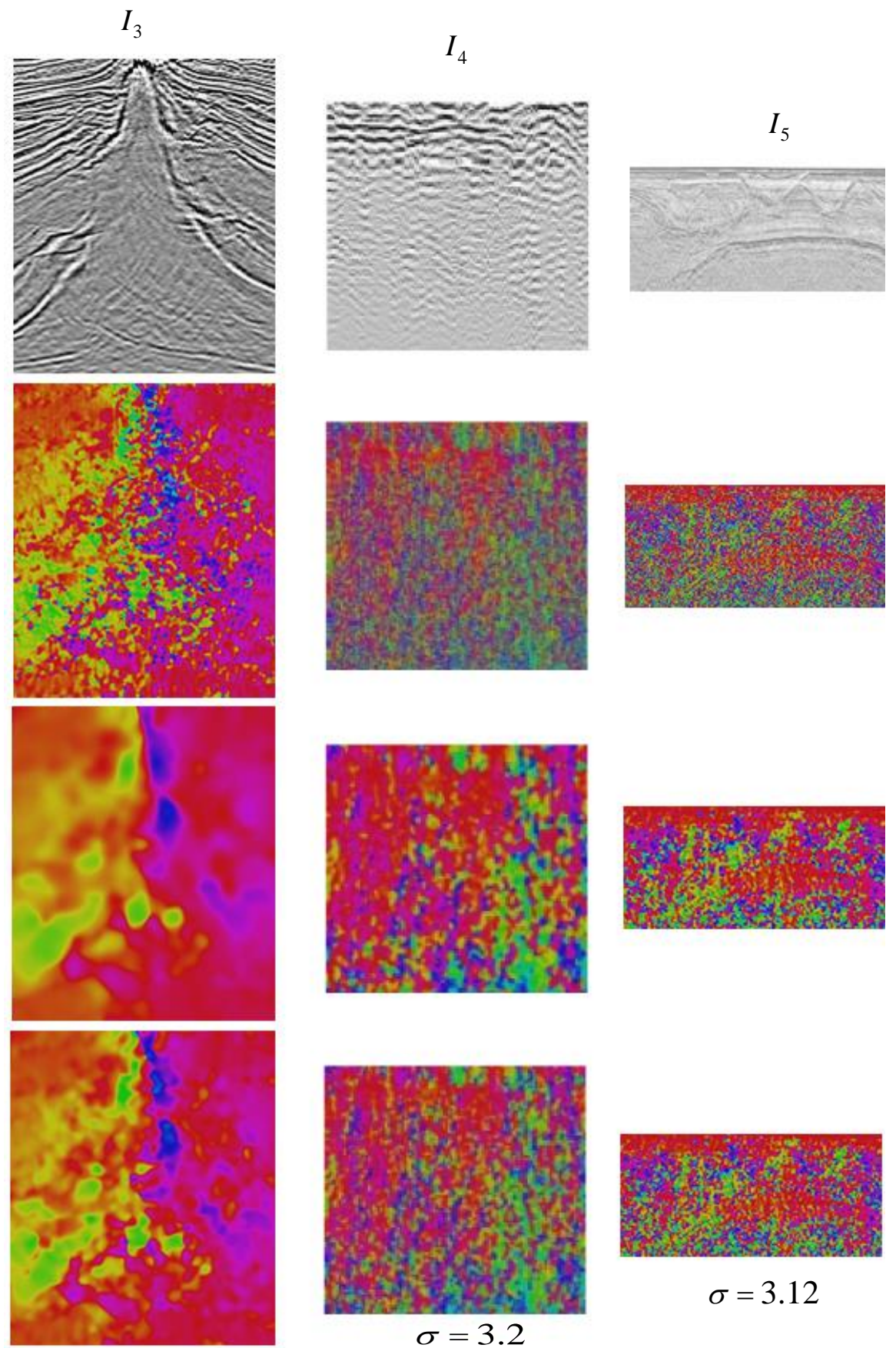

$$
\sigma=3.2
$$

$$
\sigma=3.12
$$

$\sigma=2.33$

Fig. 5. Results of the proposed algorithm on non-structured sinusoid-like textures. 1st row: the exemplars. 2nd, 3rd and 4th rows: the orientations of the matrix fields calculated using $\sigma=1, \sigma=4$ and the standard deviations obtained by the proposed algorithm 
The elongated traces oriented at $45^{\circ}$ in texture $I_{8}$ are detected as well, revealing the laminar structure at the largest scale. However, structure fluctuations are noticed in the upper-left of the orientation image. The proposed algorithm fails to capture the largest pattern in textures $I_{9}$ and $I_{10}$ due to the lack of discontinuities between the overlapping patterns, and therefore leads to irrelevant and over-smoothed matrix fields. Using a more accurate thresholding operator could be a possible solution in this case.
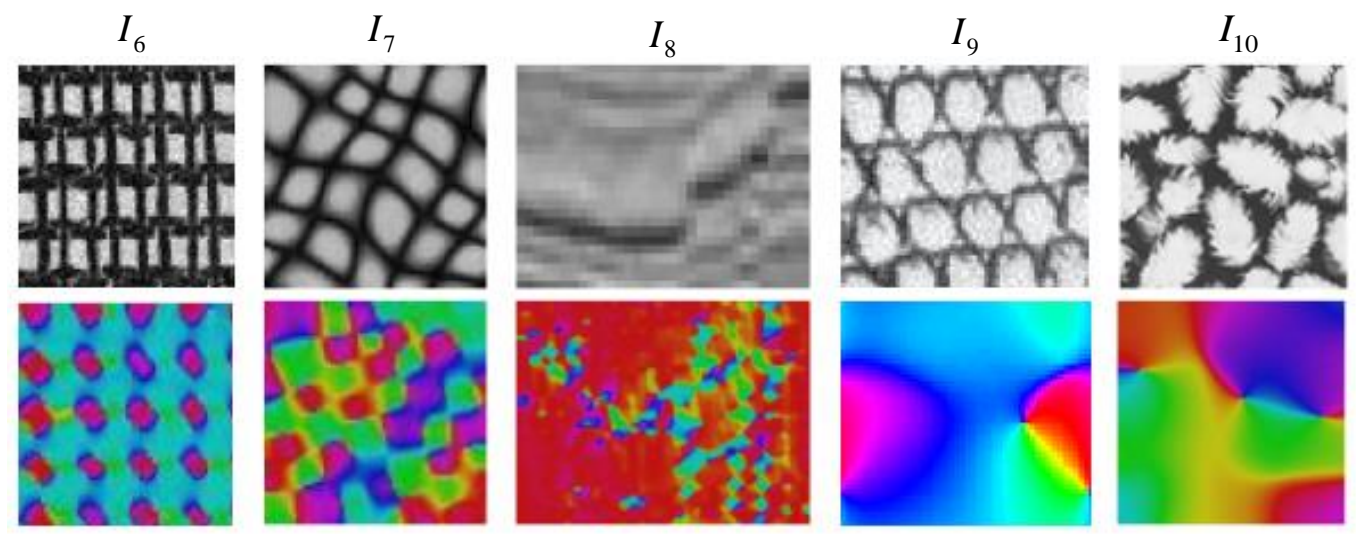

Fig. 6. Results of the proposed algorithm. 1st row: The input textures. 2nd row: The orientation image of the matrix fields calculated on the textures of the 1 st row, using a value chosen randomly from the obtained acceptable range of standard deviations

Fig. 7 presents results obtained on a set of ten textures of different types taken from Brodatz [42] database; structured, laminar, stochastic, isotropic and anisotropic. In each result, the input texture is shown on the left and the orientation image of the symmetric positive semi-definite matrix obtained by the proposed approach is on the right. It can be seen in all the results that the method succeeds in well representing the structures of the texture patterns. For instance, the periodic repetitions of the structured patterns in textures $I_{12}, I_{14}, I_{16}$ and $I_{19}$ are clearly respected. The same applies to the stochastic distribution of the patterns in textures $I_{13}, I_{15}$ and $I_{17}$ and for the laminar structures of textures $I_{11}$ and $I_{18}$. Finally, the whole set of orientations ranged between $-\pi / 2$ and $\pi / 2$, and present in texture $I_{20}$, is well detected by the positive semi-definite matrix.

Table 3 presents a comparison between the PSNR and $\mathrm{CoC}$ values obtained by the proposed approach and by the methods proposed in [14, 44, 48] using 44 textures $\left(I_{21}\right.$ to $\left.I_{64}\right)$ taken from SIPI database [49]. PSNR and CoC metrics are calculated between the coherence images of the original texture and that of a noisy version of this texture. A Gaussian noise of variance 300 is used. It can be clearly seen that in most of the results the proposed approach leads to higher PSNR values than those obtained with the other methods. This shows that the proposed algorithm succeeds in adaptively estimating the extent of gradient fields smoothing while retaining edges and shape features of the texture, which is verified by the obtained high $\mathrm{CoC}$ values. 

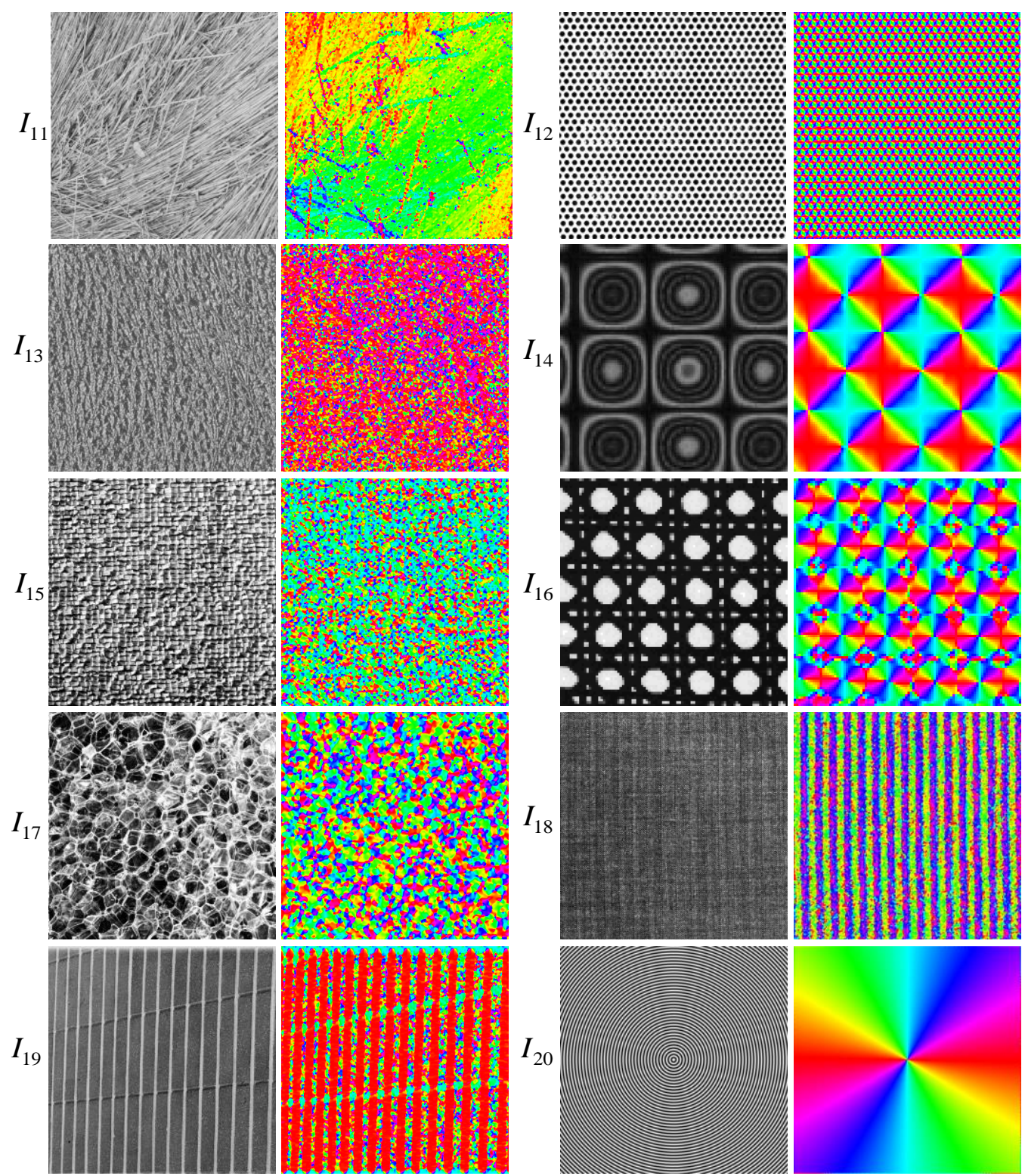

Fig. 7. Results of the proposed algorithm. In each result: Input texture (left) and orientation image obtained by the proposed approach (right)

A comparison between PSNR and CoC mertics obtained by the proposed approach and by the methods proposed in $[14,44,48]$ is summarized in the graphs of Fig. 8. The metrics are computed on the coherence images of the 115 textures of B rodatz [42] database using an added Gaussian noise of variance 300. We can clearly observe from the graphs that the PSNR objective measure depict the outperformance of the proposed algorithm with respect to the other methods, in terms of adapting the symmetric positive semi-definite matrix to the input texture. 
Table 3. PSNR and CoC values between the coherence images of SIPI textures [49] and noisy versions of these textures, obtained using the proposed approach, the methods in $[14,44,48]$

\begin{tabular}{|c|c|c|c|c|c|c|c|c|}
\hline \multirow{2}{*}{ Texture } & \multicolumn{4}{|c|}{ PSNR } & \multicolumn{4}{|c|}{$\mathrm{CoC}$} \\
\hline & Proposed & [14] & [44] & [48] & Proposed & [14] & [44] & [48] \\
\hline$I_{21}$ & 30.142 & 29.431 & 30.022 & 30.001 & 0.927 & 0.821 & 0.887 & 0.822 \\
\hline$I_{22}$ & 31.021 & 30.457 & 29.992 & 30.958 & 0.905 & 0.823 & 0.904 & 0.901 \\
\hline$I_{23}$ & 30.112 & 30.114 & 29.812 & 29.539 & 0.981 & 0.901 & 0.971 & 0.902 \\
\hline$I_{24}$ & 31.002 & 31.011 & 30.992 & 30.058 & 0.899 & 0.823 & 0.889 & 0.821 \\
\hline$I_{25}$ & 29.222 & 29.131 & 29.182 & 29.118 & 0.995 & 0.901 & 0.902 & 0.899 \\
\hline$I_{26}$ & 30.832 & 30.158 & 30.732 & 30.558 & 0.898 & 0.803 & 0.881 & 0.811 \\
\hline$I_{27}$ & 33.877 & 30.232 & 32.527 & 32.336 & 0.905 & 0.898 & 0.901 & 0.829 \\
\hline$I_{28}$ & 32.939 & 31.558 & 32.133 & 32.858 & 0.991 & 0.901 & 0.927 & 0.911 \\
\hline$I_{29}$ & 31.112 & 31.131 & 31.099 & 31.044 & 0.987 & 0.773 & 0.787 & 0.721 \\
\hline$I_{30}$ & 29.232 & 29.058 & 29.002 & 28.758 & 0.904 & 0.893 & 0.901 & 0.859 \\
\hline$I_{31}$ & 33.222 & 33.131 & 31.282 & 32.987 & 0.904 & 0.901 & 0.887 & 0.829 \\
\hline$I_{32}$ & 31.132 & 31.008 & 31.002 & 31.258 & 0.914 & 0.823 & 0.889 & 0.899 \\
\hline$I_{33}$ & 32.992 & 30.535 & 32.922 & 32.531 & 0.901 & 0.893 & 0.884 & 0.867 \\
\hline$I_{34}$ & 33.932 & 33.058 & 32.132 & 32.778 & 0.899 & 0.703 & 0.727 & 0.828 \\
\hline$I_{35}$ & 28.242 & 28.048 & 28.012 & 28.258 & 0.825 & 0.901 & 0.911 & 0.801 \\
\hline$I_{36}$ & 31.932 & 29.558 & 29.132 & 29.752 & 0.887 & 0.783 & 0.777 & 0.801 \\
\hline$I_{37}$ & 33.554 & 32.536 & 32.994 & 32.535 & 0.914 & 0.903 & 0.887 & 0.901 \\
\hline$I_{38}$ & 33.032 & 32.328 & 32.737 & 32.238 & 0.978 & 0.913 & 0.927 & 0.922 \\
\hline$I_{39}$ & 28.992 & 28.057 & 28.012 & 28.158 & 0.995 & 0.944 & 0.967 & 0.947 \\
\hline$I_{40}$ & 29.932 & 28.858 & 29.139 & 28.708 & 0.972 & 0.948 & 0.916 & 0.924 \\
\hline$I_{41}$ & 29.292 & 28.930 & 28.122 & 28.530 & 0.987 & 0.946 & 0.951 & 0.947 \\
\hline$I_{42}$ & 31.732 & 30.754 & 30.932 & 31.008 & 0.978 & 0.911 & 0.924 & 0.951 \\
\hline$I_{43}$ & 30.932 & 29.998 & 29.902 & 30.858 & 0.985 & 0.944 & 0.923 & 0.913 \\
\hline$I_{44}$ & 29.932 & 28.878 & 28.132 & 29.258 & 0.979 & 0.901 & 0.914 & 0.927 \\
\hline$I_{45}$ & 28.992 & 27.534 & 27.122 & 27.797 & 0.988 & 0.947 & 0.961 & 0.937 \\
\hline$I_{46}$ & 28.932 & 27.778 & 28.002 & 27.778 & 0.984 & 0.912 & 0.902 & 0.938 \\
\hline$I_{47}$ & 33.832 & 32.058 & 31.902 & 32.958 & 0.989 & 0.907 & 0.971 & 0.972 \\
\hline$I_{48}$ & 34.432 & 33.058 & 34.002 & 34.058 & 0.898 & 0.801 & 0.802 & 0.891 \\
\hline$I_{49}$ & 30.002 & 29.539 & 30.001 & 29.031 & 0.887 & 0.804 & 0.811 & 0.899 \\
\hline$I_{50}$ & 31.002 & 29.758 & 29.932 & 29.778 & 0.857 & 0.814 & 0.801 & 0.894 \\
\hline$I_{51}$ & 31.112 & 29.535 & 30.222 & 31.002 & 0.978 & 0.894 & 0.814 & 0.887 \\
\hline$I_{52}$ & 33.332 & 32.759 & 32.002 & 33.058 & 0.905 & 0.901 & 0.911 & 0.921 \\
\hline$I_{53}$ & 30.222 & 29.539 & 29.722 & 29.537 & 0.914 & 0.845 & 0.864 & 0.812 \\
\hline$I_{54}$ & 32.112 & 32.131 & 32.079 & 32.054 & 0.975 & 0.903 & 0.887 & 0.891 \\
\hline$I_{55}$ & 31.587 & 30.264 & 30.472 & 30.719 & 0.991 & 0.893 & 0.907 & 0.901 \\
\hline$I_{56}$ & 31.112 & 30.758 & 30.002 & 31.008 & 0.992 & 0.903 & 0.902 & 0.889 \\
\hline$I_{57}$ & 29.992 & 28.539 & 28.122 & 29.989 & 0.899 & 0.823 & 0.881 & 0.871 \\
\hline$I_{58}$ & 32.002 & 31.008 & 31.002 & 29.788 & 0.905 & 0.883 & 0.877 & 0.801 \\
\hline$I_{59}$ & 32.122 & 32.101 & 32.009 & 32.004 & 0.975 & 0.903 & 0.911 & 0.964 \\
\hline$I_{60}$ & 31.012 & 29.788 & 29.992 & 29.078 & 0.977 & 0.911 & 0.887 & 0.801 \\
\hline$I_{61}$ & 30.113 & 30.133 & 30.099 & 30.034 & 0.984 & 0.901 & 0.907 & 0.881 \\
\hline$I_{62}$ & 32.022 & 29.758 & 30.072 & 31.758 & 0.895 & 0.803 & 0.811 & 0.799 \\
\hline$I_{63}$ & 33.122 & 32.544 & 32.992 & 32.539 & 0.847 & 0.801 & 0.814 & 0.829 \\
\hline$I_{64}$ & 33.332 & 32.058 & 33.302 & 33.008 & 0.991 & 0.903 & 0.917 & 0.941 \\
\hline
\end{tabular}



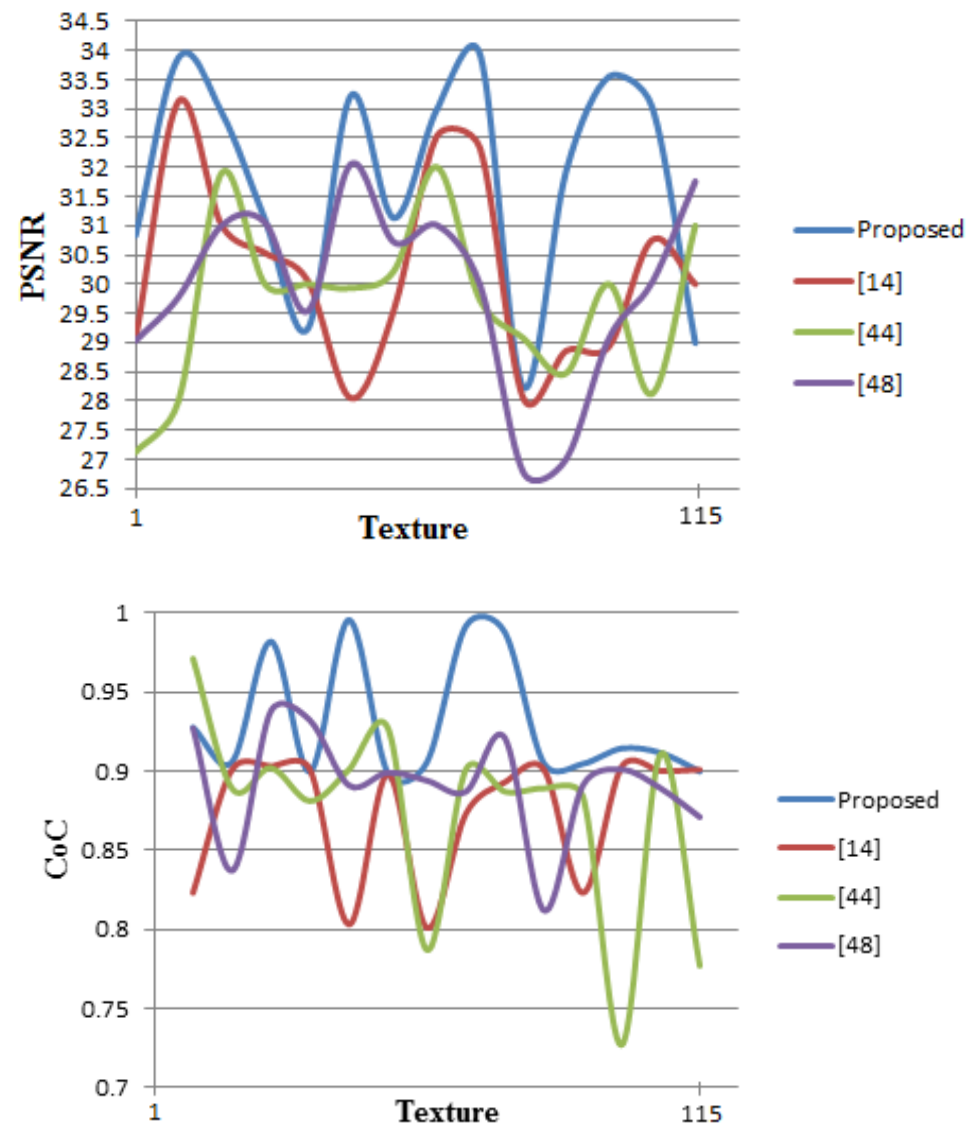

Fig. 8. PSNR and CoC values between the coherence images of B r o d a t z [42] textures and noisy versions of these textures, obtained using the proposed approach, the methods in $[14,44,48]$

This outperformance does not come at the expense of high frequency components loss, which is verified by the obtained high $\mathrm{CoC}$ metric values. In fact, the proposed approach leads to higher $\mathrm{CoC}$ values than those obtained with the other algorithms in approximately $94 \%$ of the results.

\section{Conclusions and prospects}

We have proposed a method for the size adaptation of symmetric positive semidefinite matrices, used to analyse local patterns in structured and non-structured sinusoid-like textures. In this method, the scale of the largest pattern observed in the underlying textured image is first estimated, then it is used to compute a decent size for the Gaussian weighting kernel of the matrix, leading to relevant structure layer estimation.

The influence of the weighting kernel size on the accuracy of local analysis was studied. The approach was materialized on a set of different textures. The obtained results show that even when dealing with noisy texture samples, the 
algorithm outperforms existing methods while leading to noise-free matrix fields with no over-smoothing effect, and therefore it succeeds in accurately representing the exemplar's structure.

In addition, the proposed algorithm is advantageous in automatically estimating the decent weighting kernel size, and therefore eliminating the need of repetitive matrix field computations with different values for the standard deviation of the weighting kernel.

As for future perspectives, objective evaluation metrics have to be developed. In addition, we aim at reinforcing the use of more accurate thresholding methods for a better structure extraction in textured images showing overlapping patterns.

Acknowledgments: This work has been supported by a research grant from the Higher Center for Research at the Holy Spirit University of Kaslik (USEK) and from the Lebanese National Council for Scientific Research.

\section{References}

1. Conners, R. W., C. A. H a r low. A Theoretical Comparison of Texture Algorithms. - IEEE Trans. on Pattern Analysis and Machine Intelligence, Vol. 2, 1980, pp. 204-222.

2. H a rali ck, R. M., L. G. Sh a piro. Computer and Robot Vision. Vol. 1. Addison Wesley, 1992.

3. Zhu, C. Remote Sensing Image Texture Analysis and Classification with Wavelet Transform. Zhengzhou Institute of Surveying and Mapping, Zhengzhou 450052, China, 1998.

4. Kwatra, V., A. S chöd1, I. Es s a, G. Turk, A. B obick. Graphcut Textures: Image and Video Synthesis Using Graph Cuts. - In: ACM SIGGRAPH, 2003.

5. Wikantika, K., A. Harto, R. T a te is hi. The Use of Spectral and Textural Features from Landsat TM Image for Land Cover Classification in Mountainous Area. - In: IECL Japan Workshop, Tokyo, 2001.

6. R a o, A. R. A Taxonomy for Texture Description and Identification. New York, Springer, 1990.

7. D un can, J. S., N. A y a che. Medical Image Analysis: Progress over Two Decades and the Challenges Ahead. - IEEE Trans. on Pattern Analysis and Machine Intelligence, Vol. 22, 2000, pp. 85-106.

8. Prodanov, D., T. Kon opczynski, M. Trojn ar. Selected Applications of Scale Spaces in Microscopic Image Analysis. - Cybernetics and Information Technologies, Vol. 15, 2015, No 7, pp. 5-12.

9. P e y r é, G. Texture Synthesis with Grouplets. - IEEE Trans. on Pattern Analysis and Machine Intelligence, Vol. 32, 2009, 733-746.

10. Ak1, A., C. Ya a coub, M. Donias, J. P. Da Costa, C. Germain. Structure Tensor Based Synthesis of Directional Textures for Virtual Material Design. - In: 21st IEEE International Conference on Image Processing (ICIP'14), 2014.

11. Es k e s, N., A. B o u l a n ou a r, O. F a u ger a s. Application of Image Analysis Techniques to Seismic Data. - In: IEEE International Conference on Acoustics, Speech, and Signal Processing (ICASSP'82), 1982.

12. Ak1, A., C. Ya a coub, M. Donias, J.-P. Da Costa, C. Germain. Two-Stage Color Texture Synthesis Using the Structure Tensor Field. - In: International Joint Conference on Computer Vision, Imaging and Computer Graphics Theory and Applications (VISIGRAPP'15), 2015.

13. A k 1, A., C. Y a a coub, M. D on i a s, J.-P. D a Cos t a, C. G e r m a i n. Synthèse de Texture Contrainte par Champ de Structure Arbitraire. - In: 25th Colloquium GRETSI, September 2015. 
14. A k1, A., C. Y a a coub, M. Donias, J.-P. Da Costa, C. Germain. Texture Synthesis Using the Structure Tensor. - IEEE Trans. on Image Processing, Vol. 24, 2015, No 11, pp. 4082-4095.

15. T a r t a ve 1, G., Y. G ou s s e a u, G. P e y ré. Variational Texture Synthesis with Sparsity and Spectrum Constraints. - Journal of Math. Imag. Vis., Vol. 52, 2014, No 1, pp. 124-144.

16. A g u e r r e be r e, C., Y. Gou s s e a u, G. T art a ve l. Exemplar-Based Texture Synthesis: The Efros-Leung Algorithm. - Image Process. Line, Vol. 3, 2013, pp. 223-241.

17. Galerne, B., Y. Gousseau, J.-M. Morel. Micro-Texture Synthesis by Phase Randomization. - Image Process. Line, Vol. 1, 2011 (Online).

http://dx.doi.org/10.5201/ipol.2011.ggm_rpn

18. Köppe 1, M., X. Wang, D. Dosh kov, T. Wi e gand, P. Ndjiki-Nya. Depth ImageBased Rendering with Spatio-Temporally Consistent Texture Synthesis for 3-D Video with Global Motion. - In: 19th IEEE Int. Conf. Image Process. (ICIP'12), 2012, Orlando, FL, USA, pp. 2713-2716.

19. P a g t, R., I. D. L on g st a ff. Texture Synthesis via a Non Causal Nonparametric Multiscale Markov Random Field. - IEEE Trans. on Image Processing, Vol. 7, 1998, pp. 925-931.

20. D o n a h u e, M., S. R o kh li n. On the Use of Level Curves in Image Analysis. - CVGIP Image Understanding, Vol. 1, 1993, pp. 185-203.

21. Portilla, J., E. P. S i mon celli. A Parametric Texture Model Based on Joint Statistics of Complex Wavelet Coefficients. - International Journal of Computer Vision, Vol. 40, 2000, pp. 49-71.

22. X i a n g, R., X. Z h u, F. W u, Q. X u. Object Tracking Based on Online Semi-Supervised SVM and Adaptive-Fused Feature. - Cybernetics and Information Technologies, Vol. 16, 2016, No 2, pp. 198-211.

23. Che 11 a p a, R., R. L. K a sh y a p. Texture Synthesis Using 2-D Noncausal Autoregressive Models. - IEEE Trans. on Acoustics, Speech, and Signal Processing, Vol. 33, 1985, pp. 194-203.

24. Fran cos, J. M., A. Z. Me iri, B. Por at. A Unified Texture Model Based on a 2-D WoldLike Decomposition. - IEEE Trans. on Signal Processing, Vol. 41, 1993, pp. 2665-2678.

25. Tu rn e r, M. R. Texture Discrimination by Gabor Functions. - Biological Cybernetics, Vol. 55, 1986, pp. 71-82.

26. Clark, M., A. C. Bovik, W. S. Geisler. Texture Segmentation Using Gabor Modulation/Demodulation. - Pattern Recognition Letters, Vol. 6, 1987, pp. 261-267.

27. M a 11 a t, S. Multifrequency Channel Decomposition of Images and Wavelet Models. - IEEE Trans. on Acoustic, Speech and Signal Processing, Vol. 37, 1989, pp. 2091-2110.

28. Chellappa, R., R. Ka sh y a p, B. Manjun at h. Model Based Texture Segmentation and Classification. Handbook of Pattern Recognition and Computer Vision, World Scientific Publishing, 1993, pp. 277-310.

29. C r o s s, G., A. J a i n. Markov Random Field Texture Models. - IEEE Trans. on Pattern Analysis and Machine Intelligence, Vol. 5, 1983, pp. 25-39.

30. Ge man, S., D. Geman. Stochastic Relaxation, Gibbs Distributions, and the Bayesian Restoration of Images. - IEEE Trans. on Pattern Analysis and Machine Intelligence, Vol. 6, 1984, pp. 721-741.

31. S i va ku mar, K. Morphologically Constrained GRFs: Application to Texture Synthesis and Analysis. - IEEE Trans. on Pattern Analysis and Machine Intelligence, Vol. 21, 1999, pp. $148-153$

32. Kas s, M., A. Witkin. Analyzing Oriented Patterns. - Computer Vision Graphic Image Processing, Vol. 37, 1987, pp. 362-385.

33. A k1, A., E. S a ad, C. Y a a c oub. Structure-Based Image Inpainting. - In: Proc. of 6th International Conference on Image Processing Theory, Tools and Applications, 2016.

34. A k 1, A., R. G e m a y e 1, N. A 1 k h o u r y, C. Y a a c o u b. Structure-Based Motion Estimation for Video Compresion. - In: IEEE International Multidisciplinary Conference on Engineering Technology, 2016.

35. B i g u n, J., G. G r a n 1 u n d. Optimal Orientation Detection of Linear Symmetry. - In: Proc. of 1st International Conference on Computer Vision (ICCV'87), London. Piscataway: IEEE Computer Society Press, 1987, pp. 433-438. 
36. Knutsson, H. Representing Local Structure Using Tensors. - In: 6th Scandinavian Conference on Image Analysis, 1989, pp. 244-251.

37. Jähne, B. Spatio-Temporal Image Processing: Theory and Scientific Applications. Berlin Springer-Verlag, $1993.751 \mathrm{p}$.

38. Arseneau, S., J. Cooperstock. An Improved Representation of Junctions through Asymmetric Tensor Diffusion. - In: International Symposium on Visual Computing, 2006.

39. P e r o n a, P., J. M a li k. Scale-Space and Edge Detection Using Anisotropic Diffusion. - IEEE Trans. on Pattern Analysis and Machine Intelligence, Vol. 12, 1990, pp. 629-639.

40. R a o, A. R., B. G. S chunck. Computing Oriented Texture Fields. - In: Proc. of IEEE Conference on Computer Vision and Pattern Recognition (CVPR'89), San Diego, CA, 1989, pp. 61-68.

41. A n g u 1 o, J. Structure Tensor Image Filtering Using Riemannian L1 and Lo Center-of-Mass. Image Analysis and Stereology, Vol. 33, 2014, pp. 95-105.

42. B rodatz, P. Textures: A Photographic Album for Artists and Designers. NY, USA, Dover, 1966.

43. B r ox, T., R. B o o m ga a rd, F. B. Lauze, J. We i je r, J. W e i c kert, P. M rázek, P. K o r n probst. Adaptive Structure Tensors and Their Applications. - In: Visualization and Processing of Tensor Fields. Part 1. J. Weickert and H. Hagen, Eds. Berlin, Heidelberg, Springer, 2006, pp. 17-47.

44. Touj as, V., M. Doni as, Y. B e r th o u mi e u. Structure Tensor Field Regularization Based on Geometric Features. - In: Proc. of European Signal Processing Conference (EUSIPCO'10), 2010.

45. T a n, W., T. S u n d a y, Y. T a n. Enhanced "GrabCut" Tool with Blob Analysis in Segmentation of Blooming Flower Images. - In: Proc. of International Conference on Electrical Engineering/Electronics, Computer, Telecommunications and Information Technology, 2013.

46. Munch, E., M. E. Launey, D. H. Als e m, E. Saiz, A. P. Tomsia, R. O. Ritchie. Tough, Bio-Inspired Hybrid Materials. - Science Magazine, Vol. 322, 2008, 1516-1520.

47. Donias, M., P. Baylou, N. Keskes. Curvature of Oriented Paterns: 2-D and 3-D Estimation from Differential Geometry. - In: Proc. of IEEE International Conference on Image Processing, 1998, pp. 236-240.

48. U r s, R., J.-P. D a C o s t a, J.-M. Le y s s a l e, G. Vign o le s, C. G e r m a i n. Non-Parametric Synthesis of Laminar Volumetric Textures. - In: Proc. of British Machine Vision Conference, 2012, pp. 54.1-54.11.

49. The USC-SIPI Image Database. Signal and Image Processing Institute, Ming Hsieh Department of Electrical Engineering. USC University of Southern California.

Received 14.03.2017; Second Version 15.10.2017; Accepted 20.12.2017 